\title{
Diskriminierung oder Beteiligung? Juristinnen zwischen 1933 und 1945
}

Bisher wurde die Geschichte der deutschen Juristinnen ab 1933 weitaus überwiegend als „Diskriminierungsgeschichte “ geschrieben. Im Mittelpunkt des historischen Interesses standen die Ausgrenzungsmaßnahmen des NS-Staates gegenüber den jüdischen Juristinnen sowie die Berufsbeschränkungen der nichtjüdischen Juristinnen. ${ }^{1}$ Erst kürzlich erschien der erste Beitrag, der sich auch den Karrierechancen der Juristinnen in diesen Jahren widmete. $^{2}$ Die US-Historikerin Jennifer E. Walcoff kam in diesem Beitrag gar zu dem Ergebnis, dass sich die Lage der Juristinnen in der Justiz gegenüber den letzten Jahren der Weimarer Republik kaum verändert habe. „Obwohl ihr Recht auf Planstellen als Richterinnen und Staatsanwältinnen offiziell aufgehoben war, hatte sich ihre Stellung in der Justiz de facto nur marginal verändert", schloss sie und bietet damit eine der bisherigen Deutung entgegen gesetzte These zur Geschichte der Juristinnen nach 1933 an. ${ }^{3}$ In welchem Rahmen bewegte sich also die Geschichte der Juristinnen nach 1933 zwischen Stillstand, Diskriminierung und Beteiligung?

Bereits vor 1933 mehrten sich die Stimmen unter den Vertretern der juristischen Berufe, die eine erneute Beschränkung des Zugangs für Frauen zum Richteramt usw. forderten, was nach dem Machtwechsel unter den Juristinnen zunehmend zur Verunsicherung führte. Juristinnen der älteren Generationen wehrten sich gegen die drohenden Einschränkungen ${ }^{4}$, aber auch junge und grundsätzlich mit dem nationalsozialistischen Staat politisch übereinstimmende Juristinnen begrüßten zwar einerseits den NS-Staat, nahmen aber den drohenden Ausschluss nicht widerspruchslos hin. ${ }^{5}$ Sie versuchten mit politischem und rhetorischem Geschick, ihre juristischen Tätigkeitsfelder unter Nutzung der ideologischen Vorgaben, wenn auch weitgehend vergeblich, auszuweiten und die nationalsozialistische Ideologie für ihre eigenen Ziele auszulegen. Sie betonten, die Arbeit von Rechtsanwältinnen und Richterinnen sei notwendig und insbesondere dort von Bedeutung, wo die Juristin gemäß ihres eigenen Wesens als Ergänzung zur Arbeit des Mannes die neuen Aufgaben zum Wohle des Staats erfüllen könnten. Die Juristinnen führten vor allem das Eherecht, das Jugend- und Vormundschaftsrecht sowie die Konzeption neuer Gesetze an, bei denen sie quasi als Fürsprecherin und Sprachrohr der deutschen Frau ihre Aufgaben „zum Wohl des
Volkes“ vertreten wollten. ${ }^{6}$ Diese Haltung teilte auch die Reichsfrauenführerin Gertrud ScholtzKlink, konnte sich aber damit ebenso wenig wie die Juristinnen durchsetzen. ${ }^{7}$ Die weitgehende Verdrängung der Frauen aus den juristischen Berufen

1 Vgl. den Beitrag von Simone Ladwig-Winters in diesem Heft, davor aber auch: Stefan Bajohr/Katrin RödigerBajohr, Die Diskriminierung der Juristin in Deutschland bis 1945, in: Kritische Justiz 13 (1980), S. 39-50; Deutscher Juristinnenbund, Juristinnen in Deutschland. Die Zeit von 1900 bis 2003, 4. Aufl. Baden-Baden 2003; Barbara Dölemeyer, Die Zulassung von Frauen zur Rechtsanwaltschaft und ihr Ausschluß in der NS-Zeit, in: Ascheri, Mario/Ebel, Friedrich u.a. (Hgg.), , ,Ins Wasser geworfen und Ozeane durchquert". Festschrift für Knut Wolfgang Nörr, Köln, Weimar, Wien 2003, S. 151164; Franziska-Sophie Evans von Krbek, Berufsverbot der Frauen im Dritten Reich und seine Wiedergutmachung unter der Geltung des GG, in: DRiZ 56 (1978), S. 293-298; Steffen Held, ,.... weder vor noch hinter der Barriere“, Die Verdrängung von Frauen aus den juristischen Professionen im Nationalsozialismus am Beispiel Sachsens, in: Hartmut Zwahr/Uwe Schirmer/ Henning Steinführer (Hgg.), Leipzig, Mitteldeutschland und Europa: Festgabe für Manfred Straube und Manfred Unger zum 70. Geburtstag, Beucha, 2000, S. 181191; Claudia Huerkamp, Bildungsbürgerinnen. Frauen im Studium und in akademischen Berufen 1900-1945, Göttingen 1996, S. 271-296; Anne-Gudrun Meier-Scherling, Die Benachteiligung der Juristin zwischen 1933 und 1945, in: DRiZ 1975, S. 10-13.

2 Jennifer Walcoff, Von der Staatsbürgerin zur "Volksbürgerin «: Der Disput um die Rechtsstellung der Frau, in: Steinbacher, Sybille ( $\mathrm{Hg}$.$) , Volksgenossinnen. Frauen in$ der NS-Volksgemeinschaft, Göttingen 2007, S. 48-66.

3 Walcoff, ebd., S. 66

4 Anonym, Die Frau als Juristin, in: BZ am Mittag 1933, Nr. 11, 57. Jg., 13.1.1933, o.S.; G. G., Zusammenarbeit von Mann und Frau in den juristischen Berufen, in: Die Frau 40 (1932/33), S. 312 f.; Marie-Elisabeth Lüders, Soll die Frau nur Pflichten haben? in: Vossische Zeitung v. 1.12.1932; Elisabeth Schwarzhaupt, Was hat die Frau vom Nationalsozialismus zu erwarten?, Berlin 1932.

5 Annemarie Doherr, Wille zu Einsatz und Arbeit, in: Die Frau 40 (1932/33), S. 760 f.; Edith Hegemann-Springer, Die deutsche Frau im neuen Reich, in: Europäische Revue, Sonderheft „Die Frau“, S. 494-455; dies., Wo bleiben die katholischen Frauen? in: Sonntag-Morgen, 17.9.1933, S. 3, 5

6 Ilse Eben-Servaes, Die Aufgabe der Frau als Juristin, in: Völkischer Beobachter, Norddeutsche Ausgabe, 8.2.1934, Nr. 312; dies., Die Aufgabe der Frau bei der Rechtsfindung, in: Nationalsozialistische Korrespondenz, Folge 150, 1.7.1935, S. 4; dies., Die heutige Aufgabe des weiblichen Rechtswahrers, S. 687 ff.; dies., Die Rechtswahrerin, Berlin 1941; Wiltraut von Brünneck, Die Aufgaben der Frau im Recht, in: Frauen-Kultur im Deutschen Frauenwerk, November 1937, S. 9-10; Elfriede Eggener, Die Frau im öffentlichen Leben, in: Deutsches Recht, 8 (1938), S. 94; dies., Organische Eingliederung, S. 43 f.; Adele Petmecky, Deutsche Frauen an der Arbeit. Die Juristin, in: NS-Frauenwarte, 1935, H. 21, S. 656-657.

7 Gertrud Scholtz-Klink, Die Frau im Dritten Reich, 2. Aufl. Tübingen 1998, S. 58.

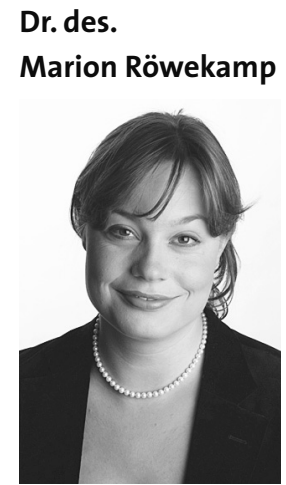

Ass. jur., Mexiko-City 
und der Juristinnen aus der Öffentlichkeit kam auch für sie als Schock, zumal die Justizverwaltungen von 1933 an bemüht gewesen waren, die Zweifel der Juristinnen und Frauen im Allgemeinen an der neuen Politik zu zerstreuen und auch nicht sofort Maßnahmen zum Ausschluss der Juristinnen erlassen hatten. ${ }^{8}$ Im Gegenteil wurden zwischen 1933 und 1935 mehr Rechtsanwältinnen zugelassen als je zuvor. Zwar deutete sich eine Einschränkung der Frau als Beamtin mit dem Gesetz vom 30. Mai 1932 über die Rechtsstellung der weiblichen Beamten bereits an, denn hier wurde in $\mathbb{} 1$ Abs. 2 festgeschrieben, dass verheiratete weibliche Beamte auch gegen deren Willen entlassen werden konnte, sofern „die wirtschaftliche Versorgung des weiblichen Beamten nach der Höhe des Familieneinkommens dauernd gesichert erscheint. "9 Dieses Gesetz wurde nach allgemeiner Rechtsauffassung im Reichsjustizministerium bis 1934 nicht auf Richterinnen angewandt. 1934 änderte sich dies jedoch im Zuge der Überlegungen zur Vereinheitlichung der Rechtspflege. Das Reichsjustizministerium holte die Meinung der Länder zur beruflichen Zukunft von Richterinnen ein. Nachdem alle für einen weitgehenden Ausschluss von Frauen plädierten, erging am 17. September 1935 aus dem

\section{Sie alle waren keine Entscheidungsträgerinnen und waren von den Verfügungen der (männlichen) Parteiführer abhängig, hatten aber in den ihnen anvertrauten weiblich definierten Arbeitsgebieten einen nicht unerheblichen Einfluss.}

Reichsjustizministerium ein Erlass, nach dem Frauen künftig nicht mehr in die Richter- und Staatsanwaltslaufbahn übernommen werden sollten. Ein Jahr später, anlässlich einer Besprechung im Reichsjustizministerium, beschloss Adolf Hitler, dass Frauen auch keine Anwältinnen mehr sollten werden können. Obwohl auch die zu diesem Zeitpunkt bereits zugelassenen Rechtsanwältinnen und die fest eingestellten Richterinnen unter diskriminierenden Maßnahmen zu leiden hatten die planmäßig angestellten Richterinnen wurden beispielsweise in weniger öffentlichkeitswirksame Positionen in den Gerichten versetzt -, wirkten diese Beschlüsse vor allem auf die Karrieren der Referendarinnen und Assessorinnen, die nun keine klassischen juristischen Berufe mehr ergreifen konnten. ${ }^{10}$

Angesichts dieser Entwicklung hatte die Regierung intern mit einer gewissen Fürsorglichkeit beschlossen, den Assessorinnen alternative Arbeits- plätze anzubieten. ${ }^{11}$ In diesem Kontext ernannte der Reichskommissar Hans Frank 1934 die Rechtsanwältin Ilse Eben-Servaes zur Sonderbeauftragten der Juristinnen und betraute sie damit, diese auf Gau- und Reichsebene neu zu organisieren. ${ }^{12}$ Assessorinnen boten sich von nun an Karrieremöglichkeiten innerhalb der NS-Frauen- oder Wohlfahrtsorganisationen: als Rechtsreferentin, als Leiterin oder Beisitzerin in den Schlichtungsstellen, als Dozentin für das deutsche Rechtsdenken in den „Führerinnenschulen“ der NS-Frauenschaft, im Reichsarbeitsdienst für die weibliche Jugend, im Stab der Reichs-Frauenführung, dem Deutschen Frauenwerk, dem BDM, dem Frauenamt der Deutschen Arbeiterfront (DAF), den studentischen Vereinigungen und der NS-Volkswohlfahrt. In diesen Funktionen waren die Assessorinnen bald so sehr gesucht, dass sogar Gedanken darüber laut wurden, wie der deutliche Rückgang der Zahl der Jurastudentinnen gestoppt werden könnte. ${ }^{13} \mathrm{Um}$ die neuen Rechtswahrerinnen zu fördern, gab es auf den Rechtswahrertagen eigene Veranstaltungen. ${ }^{14}$ Einzelne Juristinnen wie zum Beispiel die Rechtsanwältin Ilse Eben-Servaes, die nicht nur Reichsjuristinnenführerin, sondern auch in der Führung der NS-Frauenschaft sowie im Rechtswahrerbund und in der Akademie für Deutsches Recht tätig war, machten bemerkenswerte Karrieren.

Auch in der Verwaltung und in den Ministerien, wo Juristinnen in Positionen ohne Kontakt zur Öffentlichkeit arbeiten durften, standen gewisse Karrieremöglichkeiten zur Verfügung. Ein Beispiel für eine solche Karrierejuristin war Käthe Petersen, die in der Hamburger Wohlfahrtsverwaltung umfangreiche Kompetenzen an sich ziehen konnte ${ }^{15}$, oder

8 Z.B. Antwort des preußischen Justizministerium vom 12.10.1933 auf eine Anfrage von Alfred Kühnemann vom 28.9.1933, GStA Rep. 84 a Nr. 581 Fiche 12989, BI. 229.

9 RGBI. I 1932, S. 245 ff.

10 Vgl. dazu im Wesentlichen die in Fußnote 1 zitierten Literaturhinweise.

11 So z.B. Martin Bormann an das Reichsjustizministerium, 24.8.1936, BA Berlin R 43 II/427.

12 Anordnung Nr. 8/34, 10.3.1934, gez. Dr. Hans Frank, Reichjustizkommissar, BA Berlin R 3001 4182, BI. 164.

13 Die Zahl der Studentinnen der Rechtswissenschafts war von $120 \mathrm{im}$ Sommersemester 1936 auf 38 im Sommersemester 1938 zurückgegangen, s. Schreiben des Reichsgeschäftsführers des NSRB an Rechtsanwältin Dr. Eben-Servaes vom 15.6.1940, abgedruckt in: Deutscher Juristinnenbund: Juristinnen in Deutschland (1900-1989), 2. Aufl. Neuwied 1989, Anhang Nr. 31.

14 Anonym, Sondertagung der Rechtswahrerinnen, in: Deutsche Justiz 101 (1939/1), S. 896; Zur Sondertagung des Jahres 1936 vgl. Sunnus, Michael, Der NS-Rechtswahrerbund (1928-1945), Frankfurt u.a. 1990, S. 119 f.

15 Christiane Rothmaler, Die Sozialpolitikerin Käthe Petersen zwischen Auslese und Ausmerze, in: Ebbinghaus, Angela (Hg.), Opfer und Täterinnen. Frauenbiographien des Nationalsozialismus, 1987, S. 75-90. 
Marta Unger, die im Reichsministerium für Volksaufklärung und Propaganda im Juli 1934 mit der Leitung der Auslandsabteilung bei der Reichsleitung der NS-Frauenschaft und des Deutschen Frauenwerkes betraut wurde. Auch in Wohlfahrtsverbänden gab es Juristinnen, die die neue Wohlfahrtspolitik des Regimes mit trugen und effektiv den Verwaltungen zuarbeiteten. Darüber hinaus traten sie auch wissenschaftlich für eine strengere Wohlfahrtspolitik ein und prägten die Struktur und den Charakter des nationalsozialistischen Staates mit. ${ }^{16}$ Sie alle waren keine Entscheidungsträgerinnen und waren von den Verfügungen der (männlichen) Parteiführer abhängig, hatten aber in den ihnen anvertrauten weiblich definierten Arbeitsgebieten einen nicht unerheblichen Einfluss. Ähnliche Handlungsfreiheit genossen Rechtsanwältinnen wie Emma Baecker, die - ohne NSDAP Mitglied zu sein - schlicht aus Gelegenheit aus dem ihr anvertrauten Nachlass einer Jüdin Geld unterschlug, weil sie wusste, dass ihre Taten keiner strengen Kontrolle unterliegen würden. ${ }^{17}$

Alle diese Juristinnen waren Teil der nationalsozialistischen Funktionseliten. In diesen Positionen entwickelten sie Konzepte, in denen die Bedeutung des „Weiblichen” für die nationalsozialistische Rechtspflege betont wurde. Sie formten dabei weniger als die anderen Akademikerinnen den "Mutterkult” aus, sondern versuchten, auf das Familienrecht, die Wohlfahrt sowie rassen- und erbrechtliche Fragen Einfluss zu nehmen. In diesen „weiblich” definierten Bereichen konnten sie tatsächlich Entscheidungsbefugnisse und Bedeutung reklamieren und diese auch häufig weiter ausbauen. Juristinnen wie Ilse EbenServaes und die anderen in den NS-Frauenorganisationen Arbeitenden hatten darüber hinaus die wichtige Aufgabe übernommen, die NS-Ideologie geschlechtsspezifisch zu interpretieren und sie den Frauen zu vermitteln: „Wir sind gerne bereit zur Mitarbeit der Frau am neuen Staat und zur Vermittlung des nationalsozialistischen Gedankens an die deutschen Frauen“, schrieben 1933 zwei Rechtsanwältinnen an die Regierung. ${ }^{18}$ Gerade auch die Funktion der Juristinnen als Schlichterinnen innerhalb der von Ilse Eben-Servaes geleiteten Abteilung „Recht und Schlichtung“ der NS-Frauenschaft zeigt, wie sehr diese Abteilung dazu diente, die Mitglieder der NS-Frauenschaft und des Deutschen Frauenwerks und damit die deutschen organisierten Frauen zu kontrollieren und zu disziplinieren.

Was nun die These Jennifer Walcoffs angeht, dass sich jenseits der Diskriminierung der jüdischen Juristinnen die Stellung der Juristinnen in der Justiz de facto von der Weimarer Republik bis in den Nationalsozialismus hinein nicht verändert habe: sie erweist sich aufgrund der bestehenden Quellenlage als unhaltbar. Die 1935/36 von Hitler beschlossenen Maßnahmen zur Einschränkung bzw. Ausschließung der Juristinnen in und von der Justiz und der Rechtsanwaltschaft blieben trotz des kriegsbedingten erheblichen Personalmangels in der Justiz bis 1945 bestehen. Zwar waren die wenigen Richterinnen, die vor 1933 Planstellen erhalten hatten, weiterhin tätig, wie es 1936 von Adolf Hitler entschieden worden war. Auch die bis 1935 bereits zugelassenen Rechtsanwältinnen durften - solange sie nicht in andere dem NS-Regime missliebige Kategorien fielen - ihren Beruf weiterhin ausüben. Doch obwohl auch diese Berufsgruppen sich einschränken mussten, fanden die wesentlichen Beschränkungen nicht bei ihnen statt, sondern beim Juristinnennachwuchs. Keine Assessorin wurde nach 1935 zum anwaltlichen Probedienst geschweige denn zur Rechtsanwaltschaft zugelassen und es wurden keine Gerichtsassessorinnen in der Justiz planmäßig eingestellt. Das weibliche Personal in den Berufen der Rechtspflege stagnierte nicht nur auf dem Stand der Weimarer Republik, sondern begann zu sinken. Auch die von Walcoff angeführte Tatsache, dass 1940 Charlotte Granzow-Rebhuhn wieder als Richterin eingesetzt wurde ${ }^{19}$, ist kein Beweis, der ihre These zu stützen vermag. Tatsächlich gab es sogar eine Richterin, die neu ernannt wurde. ${ }^{20}$ Doch es handelte sich hier um Einzelfälle, die zu den vielen Assessorinnen und Referendarinnen, die aufgrund der Ausschlussmaßnahmen ihre Karrieren in den klassischen juristischen Berufen nicht fortsetzen oder beginnen konnten, in keinerlei Relation stehen. Es kann sich selbst unter großzügigster Schätzung nicht um mehr als zwei Prozent der Assessorinnen gehandelt haben. Die in der Weimarer Republik eingeführten Regelungen zur Zulassung der Frauen zu den originären Berufen der Rechtspflege wurden in den Jahren nach 1933 nicht nur zunichte gemacht, ${ }^{21}$ sondern die Juristinnen waren die einzige weibliche akademische Berufsgruppe, deren Ausschlussmaßnahmen durch das NS-Regime trotz der sonst bestehenden Neigung, die anfänglichen Einschränkungen in den akademischen Frauenberufen den Notwendigkeiten des Kriegs anzupassen, kaum gelockert wurden. Gleichwohl gab es auch Juristinnen, die nach 1933 in verschiedenen juristischen Bereichen tätig waren, in denen sie systemkonform mitwirkten, Karriere machten und damit zur Etablierung und Aufrechterhaltung des Nationalsozialismus beitrugen. Das Forschungsdesiderat, auch eine Geschichte der nationalsozialistischen Betätigungen von Juristinnen in den Jahren ab 1933 zu schreiben, bleibt bestehen.

16 Matthias Willing, Hilde Eiserhardt (1888-1955): Leben und Werk einer deutschen Fürsorgejuristin, Teil 2, in: NDV September 2003, S. 393-400.

17 Barbara Degen, Ein Erbe mit Hindernissen, in: Kreisverwaltung Neuwied (Hg.), Spurensuche, Johanna Loewenherz, Versuch einer Biographie, Neuwied 2008, S. $181 \mathrm{ff}$.

18 So die Rechtsanwältinnen Melitta Grimm und Gertrud Schubart an den Reichsminister des Inneren, 1.12.1933, BA Berlin R 3001/4182, BI. 162.

19 Anonym, Verheiratete weibliche Beamten, in: Die Frau 47 (1939/40), S. 379 .

20 BLHA Pr. Br. Rep. 4 a KG Berlin Pers. 2800.

21 Huerkamp, aaO., S. 287. 